PROCEEDINGS OF THE

AMERICAN MATHEMATICAL SOCIETY

Volume 136, Number 10, October 2008, Pages 3483-3492

S 0002-9939(08)09194-6

Article electronically published on May 30, 2008

\title{
PATH CONNECTIVITY OF IDEMPOTENTS ON A HILBERT SPACE
}

\author{
YAN-NI CHEN, HONG-KE DU, AND HAI-YAN ZHANG
}

(Communicated by Joseph A. Ball)

\begin{abstract}
Let $P$ and $Q$ be two idempotents on a Hilbert space. In 2005, J. Giol in [Segments of bounded linear idempotents on a Hilbert space, J. Funct, Anal. 229(2005) 405-423] had established that, if $P+Q-I$ is invertible, then $P$ and $Q$ are homotopic with $\tilde{s}(P, Q) \leq 2$. In this paper, we have given a necessary and sufficient condition that $\tilde{s}(P, Q) \leq 2$, where $\tilde{s}(P, Q)$ denotes the minimal number of segments required to connect not only from $P$ to $Q$, but also from $Q$ to $P$ in the set of idempotents.
\end{abstract}

\section{INTRODUCTION AND STATEMENT OF THE MAIN THEOREM}

Let $\mathcal{H}$ be a separable Hilbert space. The set of all bounded linear operators on $\mathcal{H}$ is denoted by $\mathcal{B}(\mathcal{H})$. An operator $P \in \mathcal{B}(\mathcal{H})$ is said to be an idempotent if $P^{2}=P$. For the sake of convenience, we denote the set of all idempotents in $\mathcal{B}(\mathcal{H})$ by $\mathcal{P}$. Two idempotents $P$ and $Q$ in $\mathcal{P}$ are said to be homotopic if they can be connected by a continuous path of idempotents in $\mathcal{B}(H)$; we shall denote this equivalence relation by $P \sim Q$. As is well known, $P \sim Q$ if and only if $\operatorname{dim} \mathcal{R}(P)=\operatorname{dim} \mathcal{R}(Q)$ and $\operatorname{dim} \mathcal{N}(P)=\operatorname{dim\mathcal {N}}(Q)$ (see [11]), where $\mathcal{R}(K)$ and $\mathcal{N}(K)$ denote the range and the null-space of an operator $K \in \mathcal{B}(\mathcal{H})$, respectively.

Recently, a number of researchers have considered questions concerning the path connectivity between idempotents (see 7, 8, 9, 11). In 1979, J. Zemánek found that the components of $\mathcal{P}$ are arcwise connected ([12]). In 1983, J. Esterle established that there exists a polynomial connection between two homotopic idempotents of $\mathcal{P}$ in a Banach algebra 8. Particularly, in 2004, J. Esterle had obtained that, for two homotopic idempotents $P$ and $Q$ in a finite dimensional real algebra, $\tilde{s}(P, Q) \leq 3$ 9. In 2005, J. Giol had proved that in an infinite dimensional Hilbert space, for two homotopic idempotents $P$ and $Q, \tilde{s}(P, Q) \leq 4$ (see [11), where $\tilde{s}(P, Q)$ denotes the minimal number of segments required to connect not only from $P$ to $Q$, but also from $Q$ to $P$ in $\mathcal{P}$. Moreover, J. Giol had proved in [1] the following result.

Theorem G ([11]). Let $P, Q \in \mathcal{P}$. If $P+Q-I$ is invertible, then $P \sim Q$ with $\tilde{s}(P, Q) \leq 2$.

Received by the editors July 18, 2006, and, in revised form, April 11, 2007.

2000 Mathematics Subject Classification. Primary 47A05, 46C07, 15A09.

Key words and phrases. Idempotent, orthogonal projection, homotopic, path connectivity.

This research was partially supported by the National Natural Science Foundation of China (10571113). 
In the present paper, our main task is to improve the above theorem by using the different ideas and methods from that in [11.

To state the main result in this note, we need a notation.

Definition 1.1. Let $P, Q \in \mathcal{P}$. If there exists an idempotent $K$ such that $\mathcal{R}(K)=$ $\mathcal{R}(P), \mathcal{N}(K)=\mathcal{N}(Q)$, then $K$ is called an insert of $P$ and $Q$, denoted by $K(P, Q)$.

Theorem 1.2. Let $P, Q \in \mathcal{P}$. Then

(1) there exists an insert $K(P, Q)$ of $P$ and $Q$ if and only if $\mathcal{R}(Q P)$ is closed, $\mathcal{R}(P) \cap \mathcal{N}(Q)=\{0\}$ and $\mathcal{N}\left(P^{*}\right) \cap \mathcal{R}\left(Q^{*}\right)=\{0\} ;$

(2) there exists an insert $K(Q, P)$ of $Q$ and $P$ if and only if $\mathcal{R}(P Q)$ is closed, $\mathcal{R}(Q) \cap \mathcal{N}(P)=\{0\}$ and $\mathcal{N}\left(Q^{*}\right) \cap \mathcal{R}\left(P^{*}\right)=\{0\}$.

The proof of this result is decomposed in Section 2. As a consequence, we get the following corollary.

Corollary 1.3. Let $P, Q \in \mathcal{P}$. Then $\tilde{s}(P, Q) \leq 2$ if and only if $\mathcal{R}(Q P)$ and $\mathcal{R}(P Q)$ are closed, $\mathcal{R}(P) \cap \mathcal{N}(Q)=\{0\}$ and $\mathcal{N}\left(P^{*}\right) \cap \mathcal{R}\left(Q^{*}\right)=\{0\}$, and $\mathcal{R}(Q) \cap \mathcal{N}(P)=\{0\}$ and $\mathcal{N}\left(Q^{*}\right) \cap \mathcal{R}\left(P^{*}\right)=\{0\}$.

From the details in Section 3, we shall see that Corollary 1.3 is an improvement of Theorem G.

\section{Proof of the main theorem and Auxiliary Results}

In this section, we begin with some notation and terminology which are used later.

Throughout this paper, the spectrum and the point spectrum, and the adjoint of $A \in \mathcal{B}(H)$ are denoted by $\sigma(A), \sigma_{p}(A), A^{*}$, respectively. An operator $P \in \mathcal{B}(\mathcal{H})$ is said to be orthogonal projection if $P^{2}=P=P^{*}$. An operator $A \in \mathcal{B}(H)$ is said to be positive if $(A x, x) \geq 0$ for all $x \in \mathcal{H}$. If $A$ is positive, then $A^{1 / 2}$ denotes the positive square root of $A$. If $\|A\| \leq 1$, then $A$ is called a contraction operator. The identity on a Hilbert space is denoted by $I$ and the restriction of the identity on a subspace $M$ of $\mathcal{H}$ is denoted by $I_{\mathcal{M}}$, or shortly by $I$ if there does not exist danger of confusion. Besides, we denote the orthogonal direct sum and topological direct sum by $\oplus$.

To prove the main result, we need some lemmas.

Lemma 2.1. Let $P, Q \in \mathcal{P}$ and $K(P, Q)$ be an insert of $P$ and $Q$. If $S$ is an invertible operator in $\mathcal{B}(H)$, then $S K(P, Q) S^{-1}$ is an insert of $S P S^{-1}$ and $S Q S^{-1}$.

Proof. Observing that $\mathcal{R}\left(S P S^{-1}\right)=S \mathcal{R}(P), \mathcal{R}\left(S K(P, Q) S^{-1}\right)=S \mathcal{R}(K(P, Q))$, by Definition 1.1, we see that $\mathcal{R}(K(P, Q))=\mathcal{R}(P)$, hence $\mathcal{R}\left(S K(P, Q) S^{-1}\right)=$ $\mathcal{R}\left(S P S^{-1}\right)$. Similarly, we also have $\mathcal{N}\left(S K(P, Q) S^{-1}\right)=\mathcal{N}\left(S Q S^{-1}\right)$. So according to Definition 1.1, $S K S^{-1}$ is an insert of $S P S^{-1}$ and $S Q S^{-1}$.

This lemma shows that an insert of two idempotents $P$ and $Q$ is invariant under similarity.

Lemma $2.2([5,10])$. Let $A \in \mathcal{B}(H)$. Then the following statements are equivalent:

(1) $\mathcal{R}(A)$ is closed.

(2) There exists an operator $X \in \mathcal{B}(H)$ such that $A X A=A$.

(3) $\mathcal{R}(A)=\mathcal{R}\left(A^{*} A\right)$. 
Lemma $2.3([2])$. Let $P, Q \in \mathcal{P}$. If $P+Q-I$ is invertible, then the following statements hold:

(1) $P(P+Q-I)=P Q=(P+Q-I) Q, Q(P+Q-I)=Q P=(P+Q-I) P$;

(2) $(P+Q-I)^{-1} P=Q(P+Q-I)^{-1}, \quad P(P+Q-I)^{-1}=(P+Q-I)^{-1} Q$;

(3) $P(P+Q-I)^{2}=P Q P=(P+Q-I)^{2} P, Q(P+Q-I)^{2}=Q P Q=$ $(P+Q-I)^{2} Q$

(4) $(P+Q-I)^{-2} P=P(P+Q-I)^{-2}, \quad Q(P+Q-I)^{-2}=(P+Q-I)^{-2} Q$.

Lemma $2.4(3)$. Let $P$ and $Q$ be orthogonal projections. If $\mathcal{R}(P) \cap \mathcal{R}(Q)=\{0\}$, $\mathcal{R}(P) \cap \mathcal{N}(Q)=\{0\}, \mathcal{N}(P) \cap \mathcal{R}(Q)=\{0\}$, and $\mathcal{N}(P) \cap \mathcal{N}(Q)=\{0\}$, then $P$ and $Q$ have the following operator matrix forms:

$$
P=\left(\begin{array}{ll}
I & 0 \\
0 & 0
\end{array}\right) \text { and } Q=\left(\begin{array}{cc}
Q_{0} & Q_{0}^{\frac{1}{2}}\left(I-Q_{0}\right)^{\frac{1}{2}} D \\
D^{*} Q_{0}^{\frac{1}{2}}\left(I-Q_{0}\right)^{\frac{1}{2}} & D^{*}\left(I-Q_{0}\right) D
\end{array}\right)
$$

with respect to the space decomposition $\mathcal{H}=\mathcal{R}(P) \oplus \mathcal{N}(P)$, respectively, where $Q_{0}$ is a positive contraction on $\mathcal{R}(P), 0$ and 1 are not in $\sigma_{p}\left(Q_{0}\right)$ and $D$ is a unitary from $\mathcal{N}(P)$ onto $\mathcal{R}(P)$.

Lemma 2.5. The set of all idempotents in $\mathcal{A}$ is invariant under similarity. That is, let $P$ be an idempotent in $\mathcal{A}$. If $S$ is an invertible element in $\mathcal{A}$, then $S^{-1} P S$ is still an idempotent.

Proof. In fact, $\left(S^{-1} P S\right)^{2}=S^{-1} P S S^{-1} P S=S^{-1} P^{2} S=S^{-1} P S$, so the result holds.

Lemma 2.6 ([7]). Let $P \in \mathcal{P}$. Then there exists an invertible operator $U \in \mathcal{B}(H)$ such that $U^{-1} P U$ is an orthogonal projection.

Lemma $2.7\left([\underline{6})\right.$. Let $A \in \mathcal{B}(H)$ be invertible and $\tilde{A}=\left(\begin{array}{cc}A & B \\ C & D\end{array}\right) \in \mathcal{B}(H \oplus K)$.

Then $\tilde{A}$ is invertible if and only if $D-C A^{-1} B$ is invertible.

Lemma 2.8 ([1). Let $P, Q \in \mathcal{P}$. The segment $[P, Q]$ is contained in the set of idempotents if and only if $(P-Q)^{2}=0$, where $[P, Q]=\{\lambda P+(1-\lambda) Q, \lambda \in[0,1]\}$.

Following we will give the proof of our main results.

Proof of Theorem 1.2. According to the property of symmetry, it is enough to show that statement (1) holds. Moreover, observing that all of the concepts considered in this theorem are similarly invariant, from Lemma 2.6, we can assume that $Q$ is an orthogonal projection.

Necessity. Define $\mathcal{H}_{1}=\mathcal{R}(P) \cap \mathcal{R}(Q), \mathcal{H}_{2}=\mathcal{R}(P) \cap \mathcal{N}(Q), \mathcal{H}_{3}=\mathcal{R}(P) \ominus\left(\mathcal{H}_{1} \oplus\right.$ $\left.\mathcal{H}_{2}\right), \mathcal{H}_{4}=\mathcal{N}\left(P^{*}\right) \ominus\left(\mathcal{H}_{5} \oplus \mathcal{H}_{6}\right), \mathcal{H}_{5}=\mathcal{N}\left(P^{*}\right) \cap \mathcal{N}(Q)$ and $\mathcal{H}_{6}=\mathcal{N}\left(P^{*}\right) \cap \mathcal{R}\left(Q^{*}\right)$. Then $P$ and $Q$ have the following operator matrix forms:

$$
P=\left(\begin{array}{cccccc}
I & & & P_{11} & P_{12} & P_{13} \\
& I & & P_{21} & P_{22} & P_{23} \\
& & I & P_{31} & P_{32} & P_{33} \\
& & & 0 & & \\
& & & & 0 & \\
& & & & & 0
\end{array}\right),
$$




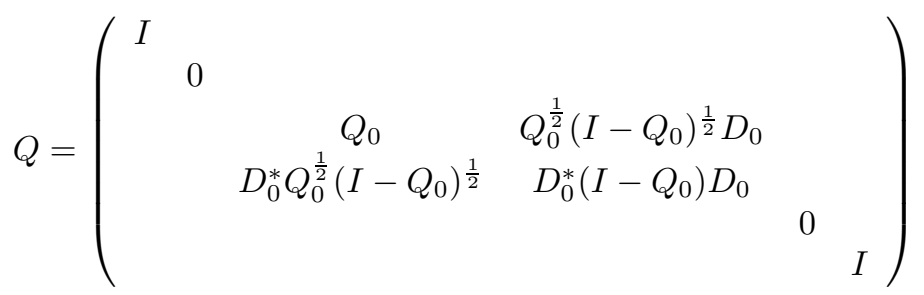

with respect to the space decomposition $\mathcal{H}=\bigoplus_{i=1}^{6} \mathcal{H}_{i}$, respectively, where $Q_{0}$ and $I-Q_{0}$ as operators defined on $\mathcal{H}_{3}$ are positive and injective, and $D_{0}$ is a unitary from $\mathcal{H}_{4}$ onto $\mathcal{H}_{3}$ by Lemma 2.4. In this case, $I-Q$ has the operator matrix

$$
I-Q=\left(\begin{array}{ccccc}
0 & & & & \\
& I & & & \\
& & I-Q_{0} & -Q_{0}^{\frac{1}{2}}\left(I-Q_{0}\right)^{\frac{1}{2}} D_{0} & \\
& -D_{0}^{*} Q_{0}^{\frac{1}{2}}\left(I-Q_{0}\right)^{\frac{1}{2}} & D_{0}^{*}\left(Q_{0}\right) D_{0} & & \\
& & & I & \\
& & & & 0
\end{array}\right) .
$$

By the assumption that there exists $K$ which is the insert of $P$ and $Q$, then $\mathcal{H}=\mathcal{R}(K) \oplus \mathcal{N}(K)=\mathcal{R}(P) \oplus \mathcal{N}(Q)$, so $\mathcal{R}(P)$ and $\mathcal{N}(Q)$ are complementary; hence $\mathcal{R}(P) \cap \mathcal{R}(I-Q)=\mathcal{R}(P) \cap \mathcal{N}(Q)=\{0\}$ by (1) and (3), that is, $\mathcal{H}_{2}=\{0\}$. From $\mathcal{H}=\mathcal{R}(P) \oplus \mathcal{N}(Q)=\mathcal{R}(P) \oplus \mathcal{R}(I-Q)$, we have $\mathcal{H}_{6}=\{0\}$ by (1) and (3), too. Now, (1), (2) and (3) can be simplified as follows:

$$
P=\left(\begin{array}{cccc}
I & & P_{11} & P_{12} \\
& I & P_{31} & P_{32} \\
& & 0 & \\
& & & 0
\end{array}\right),
$$

$$
Q=\left(\begin{array}{cccc}
I & & & \\
& Q_{0} & Q_{0}^{\frac{1}{2}}\left(I-Q_{0}\right)^{\frac{1}{2}} D_{0} & \\
& D_{0}^{*} Q_{0}^{\frac{1}{2}}\left(I-Q_{0}\right)^{\frac{1}{2}} & D_{0}^{*}\left(I-Q_{0}\right) D & \\
& & & 0
\end{array}\right)
$$

and

$$
I-Q=\left(\begin{array}{cccc}
0 & & & \\
& I-Q_{0} & -Q_{0}^{\frac{1}{2}}\left(I-Q_{0}\right)^{\frac{1}{2}} D_{0} & \\
& -D_{0}^{*} Q_{0}^{\frac{1}{2}}\left(I-Q_{0}\right)^{\frac{1}{2}} & D_{0}^{*} Q_{0} D_{0} & \\
& & & I
\end{array}\right)
$$

with respect to the space decomposition $\mathcal{H}=\mathcal{H}_{1} \oplus \mathcal{H}_{3} \oplus \mathcal{H}_{4} \oplus \mathcal{H}_{5}$, respectively.

In general, $\mathcal{H}=\mathcal{R}(P) \oplus \mathcal{R}(I-Q)=\mathcal{R}\left(P_{\mathcal{R}(P)}+I-Q\right)$ (see [10]), so $P_{\mathcal{R}(P)}+I-Q$ as a self adjoint operator is invertible, that is,

$$
P_{\mathcal{R}(P)}+I-Q=\left(\begin{array}{cccc}
I & & & \\
& 2-Q_{0} & -Q_{0}^{\frac{1}{2}}\left(I-Q_{0}\right)^{\frac{1}{2}} D_{0} & \\
& -D_{0}^{*} Q_{0}^{\frac{1}{2}}\left(I-Q_{0}\right)^{\frac{1}{2}} & D_{0}^{*} Q_{0} D_{0} & \\
& & & I
\end{array}\right)
$$


is invertible. By Lemma 2.7 and directly computing, $P_{\mathcal{R}(P)}+I-Q$ is invertible if and only if $Q_{0}$ is invertible.

The remainder of this part of the proof is to show that $\mathcal{R}(Q P)$ is closed. Observe that

$$
Q P=\left(\begin{array}{cccc}
I & & P_{11} & P_{12} \\
& Q_{0} & Q_{0} P_{31} & Q_{0} P_{32} \\
& D_{0}^{*} Q_{0}^{\frac{1}{2}}\left(I-Q_{0}\right)^{\frac{1}{2}} & D_{0}^{*} Q_{0}^{\frac{1}{2}}\left(I-Q_{0}\right)^{\frac{1}{2}} P_{31} & D_{0}^{*} Q_{0}^{\frac{1}{2}}\left(I-Q_{0}\right)^{\frac{1}{2}} P_{32}
\end{array}\right)
$$

with respect to the space decomposition $\mathcal{H}=\mathcal{H}_{1} \oplus \mathcal{H}_{3} \oplus \mathcal{H}_{4} \oplus \mathcal{H}_{5}$. Define an operator $S$ by

$$
S=\left(\begin{array}{cccc}
I & & -P_{11} & -P_{12} \\
& I & -P_{31} & -P_{32} \\
& & I & \\
& & & I
\end{array}\right)
$$

Clearly, $S$ is invertible and

$$
Q P S=\left(\begin{array}{cccc}
I & & & \\
& Q_{0} & 0 & \\
& D_{0}^{*} Q_{0}^{\frac{1}{2}}\left(I-Q_{0}\right)^{\frac{1}{2}} & 0 & \\
& & & 0
\end{array}\right) .
$$

Noting that $\mathcal{R}(Q P)=\mathcal{R}(Q P S), \mathcal{R}(Q P)$ is closed if and only if the range of the operator $\left(\begin{array}{c}Q_{0} \\ D_{0}^{*} Q_{0}^{\frac{1}{2}}\left(I-Q_{0}\right)^{\frac{1}{2}}\end{array}\right)$ is closed. For the sake of convenience, define $A=$ $\left(\begin{array}{c}Q_{0} \\ D_{0}^{*} Q_{0}^{\frac{1}{2}}\left(I-Q_{0}\right)^{\frac{1}{2}}\end{array}\right)$. Observing that
\[ A^{*} A=\left(Q_{0}, Q_{0}^{\frac{1}{2}}\left(I-Q_{0}\right)^{\frac{1}{2}} D_{0}\right)\left(\begin{array}{c}Q_{0} \\ D_{0}^{*} Q_{0}^{\frac{1}{2}}\left(I-Q_{0}\right)^{\frac{1}{2}}\end{array}\right)=Q_{0}, \]

we find that the invertibility of $Q_{0}$ implies that $\mathcal{R}(Q P)$ is closed.

Sufficiency. First, assume that $\mathcal{R}(Q P)$ is closed. Meanwhile, from the above process, we can see that $\mathcal{R}(Q P)$ is closed if and only if $Q_{0}$ is invertible.

Next, we shall construct an operator $K$ such that $K=K(P, Q)$.

Suppose that there exists an operator $K=K(P, Q)$. Since $\mathcal{R}(K)=\mathcal{R}(P), K$ should have the following operator matrix form:

$$
K=\left(\begin{array}{cccc}
I & & K_{11} & K_{12} \\
& I & K_{31} & K_{32} \\
& & 0 & \\
& & & 0
\end{array}\right)
$$

with respect to the space decomposition $\mathcal{H}=\mathcal{H}_{1} \oplus \mathcal{H}_{3} \oplus \mathcal{H}_{4} \oplus \mathcal{H}_{5}$. Since

$$
\mathcal{N}(Q)=\left\{\left(\begin{array}{c}
0 \\
-Q_{0}^{-\frac{1}{2}}\left(I-Q_{0}\right)^{\frac{1}{2}} D_{0} x_{4} \\
x_{4} \\
x_{5}
\end{array}\right): x_{i} \in \mathcal{H}_{i}, i=4,5\right\}
$$


and $\mathcal{N}(K)=\mathcal{N}(Q)$, we have $K \mathcal{N}(Q)=0$. That is,

$$
\begin{aligned}
& K\left(\begin{array}{c}
0 \\
-Q_{0}^{-\frac{1}{2}}\left(I-Q_{0}\right)^{\frac{1}{2}} D_{0} x_{4} \\
x_{4} \\
x_{5}
\end{array}\right) \\
& =\left(\begin{array}{cccc}
I & & K_{11} & K_{12} \\
& I & K_{31} & K_{32} \\
& & 0 & \\
& & & 0
\end{array}\right)\left(\begin{array}{c}
0 \\
-Q_{0}^{-\frac{1}{2}}\left(I-Q_{0}\right)^{\frac{1}{2}} D_{0} x_{4} \\
x_{4} \\
x_{5}
\end{array}\right) \\
& =\left(\begin{array}{c}
K_{11} x_{4}+K_{12} x_{5} \\
-Q_{0}^{-\frac{1}{2}}\left(I-Q_{0}\right)^{\frac{1}{2}} D_{0} x_{4}+K_{31} x_{4}+K_{32} x_{5} \\
0 \\
0
\end{array}\right)=0,
\end{aligned}
$$

hence

$$
\left\{\begin{array}{l}
K_{11} x_{4}+K_{12} x_{5}=0, \\
-Q_{0}^{-\frac{1}{2}}\left(I-Q_{0}\right)^{\frac{1}{2}} D_{0} x_{4}+K_{31} x_{4}+K_{32} x_{5}=0 .
\end{array}\right.
$$

Since $x_{4}$ and $x_{5}$ are arbitrary, then $K_{11}=0, K_{12}=0, K_{32}=0$, and $K_{31}=$ $Q_{0}^{-\frac{1}{2}}\left(I-Q_{0}\right)^{\frac{1}{2}} D$. Therefore, $K(P, Q)$ has the following matrix form:

$$
K=\left(\begin{array}{cccc}
I & & & \\
& I & Q_{0}^{-\frac{1}{2}}\left(I-Q_{0}\right)^{\frac{1}{2}} D_{0} & \\
& 0 & 0 & 0
\end{array}\right)
$$

with respect to the space decomposition $\mathcal{H}=\mathcal{H}_{1} \oplus \mathcal{H}_{3} \oplus \mathcal{H}_{4} \oplus \mathcal{H}_{5}$. In this case, noting that

$$
(P-K)^{2}=\left(\begin{array}{cccc}
0 & & P_{11} & P_{12} \\
0 & 0 & P_{31}-Q_{0}^{-\frac{1}{2}}\left(I-Q_{0}\right)^{\frac{1}{2}} D_{0} & P_{32} \\
& 0 & 0 & 0
\end{array}\right)^{2}=0
$$

from Lemma 2.8 we see that $[P, K]$ is in $\mathcal{P}$.

We will now prove that all of the points in $[K, Q]$ are idempotents, that is, $(Q-K)^{2}=0$. In fact, observing that

$$
\begin{aligned}
r c l Q-K & =\left(\begin{array}{cccc}
0 & & & \\
& Q_{0}-I & Q_{0}^{\frac{1}{2}}\left(I-Q_{0}\right)^{\frac{1}{2}} D_{0}-Q_{0}^{-\frac{1}{2}}\left(I-Q_{0}\right)^{\frac{1}{2}} D_{0} & \\
& D_{0}^{*} Q_{0}^{\frac{1}{2}}\left(I-Q_{0}\right)^{\frac{1}{2}} & D_{0}^{*}\left(I-Q_{0}\right) D_{0} & 0
\end{array}\right) \\
& =\left(\begin{array}{cccc}
0 & & & \\
& -\left(I-Q_{0}\right) & -Q_{0}^{-\frac{1}{2}}\left(I-Q_{0}\right)^{\frac{3}{2}} D_{0} & \\
& D_{0}^{*} Q_{0}^{\frac{1}{2}}\left(I-Q_{0}\right)^{\frac{1}{2}} & D_{0}^{*}\left(I-Q_{0}\right) D_{0} & \\
& & & 0
\end{array}\right),
\end{aligned}
$$

where $D_{0}$ is unitary, we get direct calculations that show that $(Q-K)^{2}=0$. 
Proof of Corollary 1.3. From (1) in Theorem 1.2, we obtain that $P$ and $Q$ can be connected by two idempotent-valued segments $[P, K]$ and $[K, Q]$; that is, $s(P, Q) \leq$ 2 , where $s(P, Q)$ denotes the minimal number of segments required to connect $P$ and $Q$ in $\mathcal{P}$. Similarly, from (2) in Theorem 1.2, we obtain $s(Q, P) \leq 2$. Therefore, $\tilde{s}(P, Q) \leq 2$.

\section{Consequences And Remarks}

Proposition 3.1. Let $P, Q \in \mathcal{P}$. If $P+Q-I$ is invertible, then $\mathcal{R}(P Q)$ and $\mathcal{R}(Q P)$ are closed, $\mathcal{R}(P) \cap \mathcal{N}(Q)=\{0\}$ and $\mathcal{N}\left(P^{*}\right) \cap \mathcal{R}\left(Q^{*}\right)=\{0\}$, and $\mathcal{R}(Q) \cap \mathcal{N}(P)=\{0\}$ and $\mathcal{N}\left(Q^{*}\right) \cap \mathcal{R}\left(P^{*}\right)=\{0\}$.

Proof. It is only to prove that $\mathcal{R}(P Q)$ is closed since $P$ and $Q$ are symmetrical.

In fact, by the assumption that $P+Q-I$ is invertible, then using Lemma 2.3, we have $P=P Q(P+Q-I)^{-1}$ and $Q=(P+Q-I)^{-1} P Q$, so

$$
P Q=P Q(P+Q-I)^{-1}(P+Q-I)^{-1} P Q=P Q(P+Q-I)^{-2} P Q .
$$

Hence $\mathcal{R}(P Q)$ is closed by Lemma 2.2 .

Meanwhile, by the fact that $P+Q-I$ is invertible, it is not difficult to get $\mathcal{R}(P) \cap \mathcal{N}(Q)=\{0\}$ and $\mathcal{R}(Q) \cap \mathcal{N}(P)=\{0\}$. Similarly, by the fact that $(P+Q-$ $I)^{*}=P^{*}+Q^{*}-I$ is invertible, we can also get that $\mathcal{N}\left(P^{*}\right) \cap \mathcal{R}\left(Q^{*}\right)=\{0\}$ and $\mathcal{N}\left(Q^{*}\right) \cap \mathcal{R}\left(P^{*}\right)=\{0\}$.

Remark 3.2. In Proposition 3.1, $\mathcal{R}((I-P)(I-Q))$ and $\mathcal{R}((I-Q)(I-P))$ are also closed. In fact, observing that $P+Q-I=-((I-P)+(I-Q)-I)$ is invertible, from Proposition 3.1 the result holds.

Remark 3.3. (1) In Theorem 1.2, the operator $P+Q-I$ is not necessarily invertible. In fact, noting that

$$
P+Q-I=\left(\begin{array}{cccc}
I & 0 & P_{11} & P_{12} \\
0 & Q_{0} & P_{31}+Q_{0}^{\frac{1}{2}}\left(I-Q_{0}\right)^{\frac{1}{2}} D_{0} & P_{32} \\
0 & D_{0}^{*} Q_{0}^{\frac{1}{2}}\left(I-Q_{0}\right)^{\frac{1}{2}} & -D_{0}^{*} Q_{0} D_{0} & 0 \\
0 & 0 & 0 & -I
\end{array}\right),
$$

then by Lemma 2.7 we see that $P+Q-I$ is invertible if and only if $Q_{0}$ and $I+Q_{0}^{-\frac{1}{2}}\left(I-Q_{0}\right)^{\frac{1}{2}} P_{31} D^{*}$ are invertible. By the discussion in the preceding section, $\mathcal{R}(Q P)$ is closed if and only if $Q_{0}$ is invertible.

This shows that our improvement is meaningful.

(2) Let $P, Q \in \mathcal{P}$. Then the fact that $\mathcal{R}(P Q)$ is closed does not imply that $\mathcal{R}(Q P)$ is closed.

For instance, define two operators $P$ and $Q$ on $\mathcal{H} \oplus \mathcal{H}$ by

$$
P=\left(\begin{array}{cc}
I & 0 \\
0 & 0
\end{array}\right) \text { and } Q=\left(\begin{array}{cc}
0 & A \\
0 & I
\end{array}\right) \text {, }
$$

respectively, where $\mathcal{R}(A)$ is not closed. It is clear that $P$ and $Q$ are idempotents. We observe that

$$
P Q=\left(\begin{array}{cc}
0 & A \\
0 & 0
\end{array}\right) \text { and } Q P=0,
$$

where $\mathcal{R}(P Q)=\mathcal{R}(A)$ is not closed, but $\mathcal{R}(Q P)$ is closed.

This shows us that the statements (1) and (2) in Theorem 1.2 are independent of each other. 
Lemma 3.4 (4]). Let $f$ be an element in a Banach algebra. If $\|1-f\|<1$, then $f$ is invertible with

$$
\left\|f^{-1}\right\| \leq \frac{1}{1-\|1-f\|}
$$

The following corollary was obtained by J. Giol in [11. Here, we give an alternative proof, which is based on the ideas and the methods used in Section 2.

Corollary 3.5 (Lemma 6.1 in [11]). The following assertions are equivalent for every pair of orthogonal projections in $\mathcal{B}(H)$ :

(1) $\|P-Q\|<1$.

(2) The element $P+Q-I$ is invertible.

(3) $\tilde{s}(P, Q) \leq 2$

(4) $\mathcal{H}=\mathcal{R}(P) \oplus \mathcal{N}(Q)$.

Proof. Let $P$ and $Q$ be a pair of orthogonal projections and denote $\mathcal{H}_{1}=\mathcal{R}(P) \cap$ $\mathcal{R}(Q), \mathcal{H}_{2}=\mathcal{R}(P) \cap \mathcal{N}(Q), \mathcal{H}_{3}=\mathcal{R}(P) \ominus\left(\mathcal{H}_{1} \oplus \mathcal{H}_{2}\right), \mathcal{H}_{4}=\mathcal{N}(P) \ominus\left(\mathcal{H}_{5} \oplus \mathcal{H}_{6}\right)$, $\mathcal{H}_{5}=\mathcal{N}(P) \cap \mathcal{N}(Q)$ and $\mathcal{H}_{6}=\mathcal{N}(P) \cap \mathcal{R}(Q)$. Then $P$ and $Q$ have the following operator matrix forms:

$$
P=\left(\begin{array}{cccccc}
I & & & & & \\
& I & & & & \\
& & I & & & \\
& & & 0 & & \\
& & & & 0 & \\
& & & & & 0
\end{array}\right)
$$

and

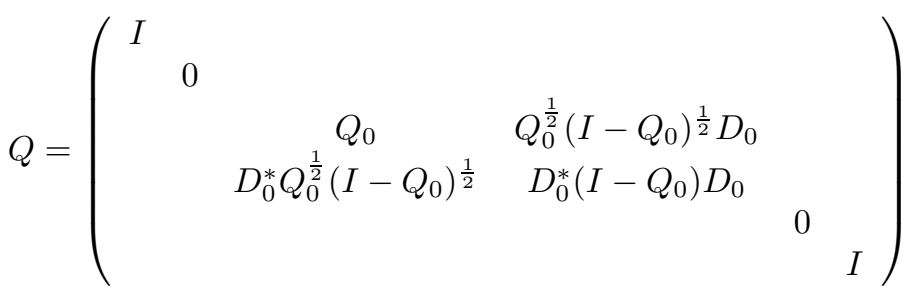

with respect to the space decomposition $\mathcal{H}=\bigoplus_{i=1}^{6} \mathcal{H}_{i}$, respectively.

Following we will complete the proof.

$(1) \Rightarrow(2)$ If $\|P-Q\|<1$, then $\mathcal{H}_{2}=\{0\}, \mathcal{H}_{6}=\{0\}$ and $\left\|I-Q_{0}\right\|<1$, so from Remark 3.3, $Q_{0}$ is invertible; hence (7) and (8) can be simplified as follows

$$
P=\left(\begin{array}{cccc}
I & & & \\
& I & & \\
& & 0 & \\
& & & 0
\end{array}\right)
$$

and

$$
Q=\left(\begin{array}{cccc}
I & & & \\
& Q_{0} & Q_{0}^{\frac{1}{2}}\left(I-Q_{0}\right)^{\frac{1}{2}} D_{0} & \\
& D_{0}^{*} Q_{0}^{\frac{1}{2}}\left(I-Q_{0}\right)^{\frac{1}{2}} & D_{0}^{*}\left(I-Q_{0}\right) D_{0} & \\
& & & 0
\end{array}\right)
$$


Observing that

$$
P+Q-I=\left(\begin{array}{cccc}
I & & & \\
& Q_{0} & Q_{0}^{\frac{1}{2}}\left(I-Q_{0}\right)^{\frac{1}{2}} D_{0} & \\
& D_{0}^{*} Q_{0}^{\frac{1}{2}}\left(I-Q_{0}\right)^{\frac{1}{2}} & -D_{0}^{*} Q_{0} D_{0} & \\
& & & -I
\end{array}\right),
$$

since $-D_{0}^{*} Q_{0} D_{0}-D_{0}^{*} Q_{0}^{\frac{1}{2}}\left(I-Q_{0}\right)^{\frac{1}{2}} Q_{0}^{-1} Q_{0}^{\frac{1}{2}}\left(I-Q_{0}\right)^{\frac{1}{2}} D_{0}=-I$ is invertible, $P+Q-I$ is invertible by Lemma 2.7 .

$(2) \Rightarrow(3)$ If $P+Q-I$ is invertible, then let $K(P, Q)=P(P+Q-I)^{-1} Q$ and $K^{\prime}(Q, P)=Q(P+Q-I)^{-1} P$, so from Theorem 1.2 , we have $\tilde{s}(P, Q) \leq 2$.

$(3) \Rightarrow(4)$ It is obvious.

$(4) \Rightarrow(1)$ Noting that $P$ and $Q$ are orthogonal projections, if $\mathcal{H}=\mathcal{R}(P) \oplus \mathcal{N}(Q)$, then $\mathcal{H}=\mathcal{R}(P) \oplus \mathcal{R}(I-Q)=\mathcal{R}(P+I-Q)$. Obviously, $P+I-Q$ is invertible, and $\mathcal{R}(P) \cap \mathcal{R}(I-Q)=\{0\}, \mathcal{R}(P) \oplus \mathcal{R}(I-Q)=\mathcal{H}$. Observe that $P$ and $I-Q$ have the following operator matrix forms:

$$
P=\left(\begin{array}{cccccc}
I & & & & & \\
& I & & & & \\
& & I & & & \\
& & & 0 & & \\
& & & & 0 & \\
& & & & & 0
\end{array}\right)
$$

and

$$
I-Q=\left(\begin{array}{cccccc}
0 & & & & & \\
& I & & & & \\
& & I-Q_{0} & -Q_{0}^{\frac{1}{2}}\left(I-Q_{0}\right)^{\frac{1}{2}} D_{0} & & \\
& & -D_{0}^{*} Q_{0}^{\frac{1}{2}}\left(I-Q_{0}\right)^{\frac{1}{2}} & D_{0}^{*} Q_{0} D_{0} & & \\
& & & & I & \\
& & & & & 0
\end{array}\right)
$$

with respect to the space decomposition $\mathcal{H}=\bigoplus_{i=1}^{6} \mathcal{H}_{i}$, respectively. In this case,

$$
\|P-Q\|=\left\|\left(\begin{array}{cc}
I-Q_{0} & -Q_{0}^{\frac{1}{2}}\left(I-Q_{0}\right)^{\frac{1}{2}} D_{0} \\
-D_{0}^{*} Q_{0}^{\frac{1}{2}}\left(I-Q_{0}\right)^{\frac{1}{2}} & -D_{0}^{*}\left(I-Q_{0}\right) D_{0}
\end{array}\right)\right\|=\left\|I-Q_{0}\right\|^{\frac{1}{2}}<1 .
$$

\section{ACKNOWLEDGMENTS}

The authors are very grateful to the referees of the manuscript for their valuable comments and suggestions which helped to improve the readability of the article and the presentation of this manuscript.

\section{REFERENCES}

[1] Y. N. Chen, H. K. Du, Idempotency of linear combinations of two idempotents on a Hilbert space, Acta. Math. Sinica 50 (2007) 1171-1176. MR2370349

[2] Y. N. Chen, H. K. Du, Y. F. Pang, A simplification of the Kovarik formula, Journal of Mathematical Analysis and its Applications 331 (2007) 13-20. MR 2305984

[3] C. Y. Deng, H. K. Du, Common complements of two subspaces and an answer to Groß's question, Acta. Math. Sinica 49 (2006) 1099-1112. MR.2285414 (2008b:47003) 
[4] R. G. Douglas, Banach Algebra Techniques in Operator Theory, Springer-Verlag, New York, 2003. MR.1634900 (99c:47001)

[5] H. K. Du, C. Y. Deng, The representation and characterization of Drazin inverses of operators on a Hilbert space, Linear Algebra Appl. 407 (2005) 117-124. MR2161918 (2006d:47001)

[6] H. K. Du, X. Y. Yao, C. Y. Deng, Invertiblity of linear combinations of two idempotents, Proc. Amer. Math. Soc. 134 (2006) 1451-1457. MR2199192 (2006k:47004)

[7] H. K. Du, W. F. Wang, Y. T. Duan, Path connectivity of $k$-generalized projectors, Linear Algebra Appl. 422 (2007) 712-720. MR2305151 (2008b:47004)

[8] J. Esterle, Polynomial connections between projections in Banach algebras. Bull. London Math. Soc. 15 (1983) 253-254. MR697127 (84g:46069)

[9] J. Esterle, J. Giol, Polynomial and polygonal connections between idempotents in finitedimensional real algebras, Bull. London Math. Soc. 36 (2004) 378-382. MR 2038725 (2005b:46100)

[10] L. R. Fillmore, J. P. Williams, On operator ranges, Advances in Math. 7 (1971) 254-281. MR0293441 (45:2518)

[11] J. Giol, Segments of bounded linear idempotents on a Hilbert space, J. Funct. Anal. 229 (2005) 405-423. MR2182594 (2006h:47062)

[12] J. Zemánek, Idempotents in Banach algebra, Bull. London Math. Soc. 11 (1979) 177-183. MR0541972 (80h:46073)

Department of Mathematics, Shaanxi University of Technology, Hanzhong 723001, People's Republic of China

E-mail address: operatorguy@126.com

College of Mathematics and Information Science, Shaanxi Normal University, Xi'an 710062, People's Republic of China

E-mail address: hkdu@snnu.edu.cn

College of Mathematics and Information Science, Shaanxi Normal University, Xi'an 710062, People's Republic of China 